\title{
Autonomous and Body Weight Suppressing Exoskeleton to Support Human Walk
}

\author{
Nazim Mir-Nasiri \\ Nazarbayev University \\ 53, Kabanbay Batyr Avenue, Astana, Kazakhstan \\ mir-nasiri.nazm@nu.edu.kz
}

\section{Extended Abstract}

During the last decade, researchers have focused on the development of lower limb exoskeletons that are now applied to several fields, including power augmentation for the military [1], medical assistance [2], and rehabilitation [3], [4].

The novelty of the design is that it presents an exoskeleton structure that is able to interact with the human via flexible sensors such as strain gauges. The gauges are in touch with the thigh and shank of human legs. There are also pressure sensors that are in touch with the human feet. The strain gauges sense the human intention to move the legs during the walk and the pressure sensors detect the moment of highest pressure of the body weight on the feet during the walk. The exoskeleton is tight up with the human legs by means of flexible cuffs and has a narrow seat in between the human legs. Each leg of the exoskeleton structure has four degrees of freedom; two of them at the human hip level (sagittal and transverse joints) and each one at each human knee and ankle level (flexion joints). Out of 4 degrees of freedom only two (at the knee and hip) are motor driven. The rest of degrees are freely driven by human himself during the walk.

The advantage and uniqueness of the system as compared to the existing exoskeletons is in its ability to support automatically the weight of the human body while the subject moves in forward direction. It has unique ability to decouple the weight/mass carrying function of the system from its forward motion function. This ability makes the exoskeleton design more efficient by reducing its power consumption, weight and size of the propulsion motors and saves the power of batteries for a longer run before the recharging. The weight support takes place when the system senses the predetermined level of pressure at the exoskeleton foot. Normally it happens when the object is standing on one leg and swings the other one. At that instant the knee motor of the standing leg is deactivated by the controller and the motion at the knee is blocked by the passive pneumatic cylinder that is located across the knee. The leg becomes a rigid structure. As a result the human passes this stage of walk cycle just by sitting on the seat and not by contracting much its leg muscles (motion assistance). In order to maintain the rigid conditions of the exoskeleton the human still needs to apply a predefined pressure on the exoskeleton foot. This condition would serve well for the gradual rehabilitation of leg muscles of those who had leg injuries (medical assistance). The control of the exoskeleton operations is done automatically by PID close-loop controller that receives the signals from all the sensors and provides respective input signals to the motor drivers and the valves of pneumatic cylinders across the knee joints. The prototype of the system is being currently developed.

\section{References}

[1] A. B. Zoss, H. Kazerooni, and A. Chu, "Biomechanical design of the Berkeley lower extremity exoskeleton (BLEEX)," IEEE/ASME Transactions on Mechatronics, vol. 11, no. 2, pp. 128-138, 2006.

[2] K. Suzuki, G. Mito, H. Kawamoto, Y. Hasegawa, and Y. Sankai, "Intention-based walking support for paraplegia patients with robot suit HAL," J. Advanced Robotics, vol. 21, pp. 1441-1469, 2007.

[3] Y. Mao, S. K. Agrawal, "Design of a Cable Driven Arm Exoskeleton (CAREX) for Neural Rehabilitation," IEEE Transactions on Robotics, vol. 28, no. 4, pp. 922-931, 2012.

[4] J. Iqbal and K. Baizid, "Stroke rehabilitation using exoskeleton-based robotic exercisers Mini Review," $J$. Biomedical Research, vol. 26, no. 1, pp. 197-201, 2015. 